\title{
Hablando de emociones fingidas: la relación entre la sintaxis de la complementación y la comprensión de la simulación emocional
}

\author{
Francesc Sidera-Caballero*, Anna Amadó-Codony y Elisabet Serrat-Sellabona
}

Universitat de Girona

\begin{abstract}
Resumen: Este trabajo se centra en investigar la relación entre el uso de la sintaxis de la complementación y la capacidad infantil para comprender la simulación emocional. Un total de 337 niñas y niños de cuatro a doce años realizaron cuatro tareas en las cuales los personajes fingían una emoción, para jugar o para engañar a un observador. Se preguntó a los participantes por la emoción externa e interna del protagonista, y también por las creencias del observador, solicitando la justificación de sus respuestas. Se analizaron tanto la capacidad para comprender las emociones fingidas como el uso de distintos tipos de oraciones completivas en las justificaciones de las respuestas. Los resultados mostraron una contingencia significativa entre la comprensión de la simulación emocional y la utilización de oraciones completivas con verbos cognitivos o comunicativos, aunque el uso de este tipo de oraciones no se mostró necesario ni suficiente para esta comprensión. Finalmente, se discute el alcance de la relación entre la comprensión de la simulación emocional y la utilización de oraciones completivas con verbos comunicativos o cognitivos.

Palabras clave: Oraciones completivas; simulación emocional; distinción apariencia-realidad; engaño; juego simbólico.
\end{abstract}

Title: Talking about pretend emotions: the relation between the syntax of complementation and the understanding of emotional simulation

Abstract: This work aims at investigating the relationship between the use of the syntax of complementation and children's ability to understand emotional simulation. A total of 337 children from four to twelve years of age were administered four tasks in which the characters simulated an emotion, either to play or to deceive an observer. Participants were asked both about the external and the internal emotion of the protagonist, and also about the beliefs of the observer. Children were requested to justify their responses. Children's ability to understand simulated emotions and their use of different types of sentential complement sentences were analysed. The results uncovered a significant contingency between children's understanding of simulated emotions and their use of sentential complement sentences with cognitive or communicative verbs. However, the use of this type of sentences was not found necessary or sufficient for children to understand emotional simulation. The extent of the relationship between the understanding of emotional simulation and the use of sentential complement sentences is discussed.

Key words: Sentential complement sentences; emotional simulation; appearance-reality distinction; deception; pretend play.

\section{Introducción}

El objetivo principal de la presente investigación es estudiar la relación entre un aspecto concreto del lenguaje, las oraciones completivas, y la capacidad de los niños para distinguir entre la emoción externa y la emoción interna de una persona (o la comprensión de la simulación emocional), en situaciones de engaño y en situaciones de juego simbólico. La comprensión de esta distinción es importante porque permite comprender que las personas podemos esconder $\mathrm{o}$ fingir emociones, y que esto puede tener consecuencias en las creencias de los demás sobre nuestros sentimientos. Esta es una de las capacidades que constituyen la denominada teoría de la mente (en adelante, TM).

Se entiende por TM la capacidad de las personas para comprender los estados mentales internos (creencias, actitudes, emociones, conocimiento, puntos de vista...) propios y ajenos. Además, de acuerdo con de Villiers (2007), esta capacidad permite explicar y predecir el comportamiento de las personas a partir de la inferencia de dichos estados mentales. Esta capacidad de comprensión sociocognitiva parece depender del desarrollo del lenguaje y, a su vez, facilitarlo (Resches, Serrat, Rostan y Esteban, 2010). En este sentido, varias investigaciones actuales han intentado desmenuzar la relación de interdependencia entre el desarrollo del lenguaje y la TM (ver, por ejemplo: Astington y Baird, 2005; de Villiers, 2007; Miller, 2006; Milligan, Astington y Dack, 2007;

* Dirección para correspondencia [Correspondence address]: Francesc Sidera Caballero. Facultat d'Educació i Psicologia. Campus de Barri Vell. Plaça Sant Domènec, 9, 17071 Girona (España). E-mail: francesc.sidera@udg.edu.
Slade y Ruffman, 2005). Algunos autores, como, por ejemplo, Astington y Jenkins (1999), han destacado el papel especial de la sintaxis en el desarrollo de la TM y otros, como, por ejemplo, de Villiers (2005; 2007), han especificado que son las oraciones completivas las que tienen un papel crucial en este desarrollo. Cómo veremos más adelante, el argumento de de Villiers se basa en el hecho de que en algunas oraciones completivas, como por ejemplo en la frase "la niña piensa que el coche es rojo", aunque la oración subordinada sea falsa, la oración principal sigue siendo verdadera. De este modo, este tipo de estructuras, argumenta de Villiers, ofrecen el formato necesario para representar el hecho de que las persones podemos tener representaciones erróneas sobre la realidad, o falsas creencias. Esta comprensión de la falsa creencia ha sido considerada, por muchos autores, como crucial en el desarrollo de la TM (Wellman, Cross y Watson, 2001).

El desarrollo de la sintaxis de la complementación en niños comienza a producirse entre su segundo y tercer aniversario (Bloom, Rispoli, Gartner y Hafitz, 1989; Limber, 1973), aunque con grandes restricciones de función y forma (Kidd, Lieven y Tomasello, 2005). De acuerdo con Kidd, Lieven y Tomasello (2006), el conocimiento temprano de las oraciones completivas incluye un rango limitado de verbos y es específico para cada uno de ellos. Para aprender oraciones completivas con nuevos verbos, según estos autores, será importante tanto la frecuencia léxica como la complejidad conceptual del verbo. En el caso de la lengua inglesa, parece ser que los niños empiezan a producir oraciones completivas con verbos cognitivos alrededor de los 4 años (Stephens, 2006). Sin embargo, dado que las estructuras sintácticas varían de una lengua a otra, es posible que haya diferencias en el momento de adquisición de las oraciones completivas en 
función de la lengua. Tal como comentan Cheung et al. (2004), las aserciones sobre los efectos de la comprensión de la complementación deben ser verificadas en lenguas con diferente estructura de complementación. Katis y Stampouliadou (2009), por ejemplo, en un estudio de caso en lengua griega, observaron construcciones con el verbo 'pensar' referidas a falsas creencias desde antes de los 3 años. En el caso de la lengua catalana, que es la utilizada por los sujetos del presente estudio, los niños comienzan a utilizar oraciones completivas con el verbo querer (verbo volitivo) alrededor de los dos años y medio (Aparici, Serrat, Capdevila y Serra, 2001). Sin embargo, según de Villiers (2005), aunque los niños sean capaces de utilizar oraciones completivas en su habla espontánea con anterioridad, no es hasta los 4 años de edad cuando pueden dominar las estructuras de complementación. A continuación, se revisarán algunos estudios centrados en la relación entre la sintaxis de la complementación y la TM.

Como se ha argumentado antes, de Villiers (2005; 2007) propone que el dominio de la sintaxis de la complementación provee de un formato representacional necesario para razonar sobre el pensamiento de los demás que no es posible con otras formas de representación. Otros autores, como Jackendoff (1996) y Segal (1998), argumentan incluso que este tipo de lenguaje es imprescindible para poder razonar sobre la falsedad o veracidad del contenido de la mente de los demás. De Villiers (2005) propone, sin embargo, que, dentro de las oraciones completivas, sólo las del tipo realis con verbos cognitivos y comunicativos permiten el tipo de razonamiento implicado en la comprensión de las creencias falsas de los demás, aspecto primordial de la TM. En efecto, de Villiers y de Villiers (en prensa) hallaron que la sintaxis de la complementación con verbos comunicativos explicaba mejor la comprensión de los niños de la falsa creencia que el vocabulario o la sintaxis general. Además, algunos estudios longitudinales han mostrado que el dominio de la sintaxis de la complementación es un predictor de la futura comprensión de la falsa creencia, tanto en niños con desarrollo normal (de Villiers y Pyers, 2002), como en niños con autismo (Lind y Bowler, 2009; Tager-Flusberg y Joseph, 2005), deficiencia auditiva (Schick, de Villiers, de Villiers y Hoffmeister, 2007) o trastorno específico del lenguaje (de Villiers, Burns y Pearson, 2003; Miller, 2004). Además, varios estudios han postulado que la contribución de la sintaxis en la comprensión de la TM supera a la del léxico, tanto en niños con desarrollo normal (Astington y Jenkins, 1999) como en niños con trastornos del espectro autista (Fisher, Happé y Dunn, 2005; Paynter y Peterson, 2010). Por otra parte, en un metaanálisis sobre la relación entre la sintaxis de la complementación y la TM, Milligan et al. (2007) hallaron un efecto más fuerte de las medidas de lenguaje individual hacia la falsa creencia que a la inversa, obteniendo el dominio de las estructuras de complementación el mayor tamaño del efecto. Low (2010) destaca, en cambio, que esta medida del efecto podría ser exagerada y que el razonamiento sobre la falsa creencia correlaciona de forma similar con otras medidas de la habilidad lingüística. Al mismo tiempo, el estudio de Low pone de manifiesto que la relación entre el dominio de la sintaxis de la complementación es evidente para el éxito en tareas de falsa creencia explícita pero no en tareas que evalúan la comprensión implícita (como las tareas que utilizan la dirección de la mirada como respuesta). Otros autores también dudan de la importancia primordial de la sintaxis en el desarrollo de la TM. Cheung et al. (2004), por ejemplo, no obtuvieron ninguna relación entre la comprensión de la sintaxis de la complementación per se y la TM, ni en inglés ni en cantonés, considerando la comprensión general del lenguaje como un factor más importante a tener en consideración. Similarmente, Slade y Ruffman (2005) no hallaron ninguna evidencia a favor de que la sintaxis tuviera un papel más importante que la semántica en la contribución del lenguaje en TM, sugiriendo que ambas favorecen la comprensión de la falsa creencia. Perner, Sprung, Zauner y Haider (2003) argumentan que la sintaxis no puede ser la causa del progreso en TM, porque en la lengua alemana los verbos de deseo y de creencia tienen una estructura de complemento similar y, sin embargo, los niños pueden responder a preguntas de deseo antes que a preguntas de creencia. A este respecto, J. de Villiers (2005) defiende que lo crucial no es sólo la semántica del verbo sino también la proposición que éste tiene incrustada.

Por otro lado, algunos estudios de entrenamiento han mostrado la eficacia de una intervención basada en el uso de oraciones completivas para desarrollar el razonamiento sobre falsas creencias (Hale y Tager-Flusberg, 2003; Lohmann y Tomasello, 2003; aunque ver Miller y Verrilli, 2005). No obstante, como sugieren Serrat et al. (en prensa), los entrenamientos en oraciones completivas podrían ser solo eficaces para fomentar la comprensión de la falsa creencia cuando estas oraciones se refieren a realidades con más de una perspectiva, como en el caso de los objetos engañosos. Este hallazgo coincide con la propuesta de Papafragou, Cassidy y Gleitman (2007), que resaltan la importancia de las situaciones de falsa creencia, en contraposición a las de creencia cierta, para el aprendizaje de los verbos de estado mental. Por otra parte, Harris (2005) comenta que el diálogo que enfatiza diferentes puntos de vista para un mismo evento u objeto es suficiente para promover la resolución de tareas de TM. De este modo, este autor relativiza la importancia del dominio de las estructuras de complementación. Una vez revisados los estudios centrados en la relación entre la sintaxis de la complementación y la TM, se consideró que estas estructuras sintácticas son importantes para la comprensión de las creencias falsas, aunque quizás no imprescindibles. En esta línea, Remmels y Peters (2009) hallaron que aunque la mayoría de niños de su estudio dominaban la sintaxis de la complementación, aun se equivocaban en la resolución de tareas de falsa creencia. Así pues, todavía es necesario profundizar en la relación entre la sintaxis de la complementación y las tareas de falsa creencia. Como hemos introducido, el presente estudio se centra en la relación entre la sintaxis de la complementación y la comprensión de la simulación 
emocional, aspecto que, como veremos, implica comprender las creencias falsas. Por ello, a continuación se presentarán algunos estudios que describen la capacidad infantil para comprender que las emociones aparentes y reales pueden ser diferentes, y se argumentará la posible relación de esta comprensión con el dominio de la sintaxis de la complementación.

La capacidad para comprender que las emociones que expresan las personas pueden ser distintas de cómo éstas se sienten es un hito importante en el desarrollo de la TM, porque permite a los niños comprender que las personas pueden ocultar sus emociones a los demás (Bretherton, Fritz, Zahn-Waxler, y Ridgeway, 1986). Harris, Donnelly, Guz, y Pitt-Watson (1986) realizaron un estudio pionero en el que presentaban a los niños historias donde el protagonista tenía un motivo para ocultar sus emociones a un observador (tareas de apariencia-realidad (AR) emocional). Los participantes debían demostrar su comprensión de que las emociones externas del protagonista podían ser diferentes de sus emociones internas. Las investigaciones realizadas desde entonces, sugieren que esta comprensión comienza a desarrollarse en situaciones de engaño a partir de los 4 años (Gross y Harris, 1988; Harris et al., 1986; Wellman y Liu, 2004), se sistematiza en torno a los 6 años, y sigue mejorando hasta, al menos, los 10 u 11 años (Pons, Harris, y de Rosnay, 2004). El estudio acerca de que las emociones fingidas en situaciones de ficción no son reales no ha recibido tanta atención; sin embargo, el estudio de Sidera, Serrat, Rostan, y SanzTorrent, (2011) sugiere que a los 4 años los niños no son capaces de darse cuenta de que cuando alguien finge una emoción, en realidad se puede sentir diferente. Según estos autores, esta comprensión empezaría, al menos, de forma explícita, en torno a los 6 años.

Para comprender que las emociones que se muestran pueden diferir de las internas, es necesaria la comprensión de la falsa creencia (Peterson y Wellman, 2009; Wellman y Liu, 2004), hecho que ocurre alrededor de los 3 o 4 años de edad (Hansen, 2010; Wellman, Cross, y Watson, 2001). El mismo requisito ha sido argumentado para la comprensión de que las emociones fingidas pueden tener consecuencias en las creencias de los observadores (Gross y Harris, 1988). Por tanto, si es cierto que las oraciones completivas tienen un papel importante en la comprensión de la falsa creencia, como sugieren algunos de los estudios anteriormente mencionados, se puede suponer que estas oraciones también tienen un papel importante en la comprensión de la distinción entre la emoción externa y emoción interna (en adelante, comprensión AR emocional). Con el objetivo de profundizar en el conocimiento de esta relación, se presentaron a los niños historias de engaño o de juego de ficción, donde los protagonistas simulaban emociones, para comprobar si los niños que resolvían correctamente las tareas de AR emocional utilizaban oraciones completivas en sus respuestas.

Teniendo en cuenta que algunos estudios han encontrado que la sintaxis de la complementación no era una condición suficiente para resolución de tareas de falsa creencia
(Remmels y Peters, 2009), y dado que las tareas de AR emocional se comprenden más tarde que las tareas de falsa creencia (Wellman y Liu, 2004), nuestra primera hipótesis es que algunos niños del presente estudio utilizarán oraciones completivas con verbos comunicativos o cognitivos y aun así no comprenderán la distinción AR emocional. Por otro lado, dado que el dominio de la sintaxis de la complementación parece una condición, aunque no suficiente, sí importante para la comprensión de las creencias falsas, nuestra segunda hipótesis es que los niños que hayan adquirido la comprensión AR emocional utilizarán oraciones completivas con verbos cognitivos o comunicativos en sus respuestas.

El hecho de considerar este tipo de verbos en nuestras hipótesis se justifica a partir de de Villers (2005), quien afirma que las oraciones completivas con este tipo de verbos proveen del formato representacional para comprender las creencias falsas. Por otra parte, para valorar la comprensión de la AR emocional se consideraron dos tipos de historias: de engaño y de juego de ficción. La razón de ello, tal como se explica en el estudio de Sidera, Serrat, Rostan y SanzTorrent (2011) fue investigar posibles diferencias entre ambas historias en relación con la comprensión AR emocional. En dicho estudio se halló una mayor comprensión de la AR emocional en las situaciones de engaño que en situaciones de juego de ficción, de acuerdo con los autores debido a los motivos de los personajes para esconder una emoción. Por este motivo, se optó por incorporar esta variable. En este sentido, se planteó explorar si la relación entre la comprensión de la AR emocional y el uso de las oraciones completivas se mostraba igual en ambas condiciones. Finalmente, dado que se pretendía estudiar la relación entre el desarrollo de la comprensión AR emocional y la utilización de oraciones completivas, la edad también se incluyó como variable independiente.

\section{Método}

\section{Participantes}

La muestra estaba formada por 162 niñas y 175 niños provenientes de 6 centros públicos de educación infantil y primaria y de un instituto de educación secundaria, también público, de la ciudad de Girona. Los participantes se dividieron en 5 grupos en función de su edad cronológica: un grupo de 4 años, con 64 participantes (Rango: 4;0 a 4;6; Media: 4:3; 36 niñas), un grupo de 6 años, con 73 participantes (Rango: 6;0 a 6;6; Media: 6;3; 35 niñas), un grupo de 8 años, de 64 participantes (Rango: 8;0 a 8;6; Media: 8;3; 30 niñas), un grupo de 10 años, con 72 participantes (Rango: 10;0 a 10;6; Media: 10;3; 30 niñas), y un grupo de 12 años, de 64 participantes (Rango: 12;0 a 12;6; Media: 12;3; 31 niñas).

\section{Material}

Para llevar a cabo el estudio se diseñaron 8 tareas: 4 tareas en la condición de juego simbólico y 4 en la condición de 
engaño. En cada tarea se presentaron 2 dibujos de 15 x 21 $\mathrm{cm}$. En el Apéndice 1 se muestran ejemplos de las tareas utilizadas. Todas las tareas seguían el mismo esquema. En el Dibujo 1 se explicaba a los participantes la situación real del protagonista, que provocaba su emoción interna. En el Dibujo 2 se explicaba que el protagonista se inventaba una situación imaginaria y simulaba una emoción, mostrando, a través de la expresión facial, la emoción opuesta a la que mostraba en el Dibujo 1. En dos de las tareas de cada condición, la emoción interna era positiva (el protagonista estaba contento) y en las otras dos tareas la emoción interna era negativa (el protagonista estaba triste).

En las tareas de juego simbólico, el protagonista inventaba una situación imaginaria y proponía a un observador el juego de fingir una realidad alternativa. Para hacer esta propuesta, el protagonista utilizaba una oración completiva con la locución verbal "hacer que" o "hacer ver que" (fer veure que en catalán).

En las tareas de engaño, el protagonista decía una mentira para engañar a un observador. En estas tareas se utilizaban oraciones completivas con los verbos "pensar" y "querer".

Se utilizó una grabadora digital de audio para registrar las respuestas de los niños, que fueron transcritas para ser analizadas posteriormente.

\section{Procedimiento}

Cada participante fue asignado aleatoriamente a una de las 2 condiciones experimentales, de juego simbólico o de engaño. Dentro de cada grupo de edad, aproximadamente la mitad de los participantes fueron asignados a una condición y la otra mitad a la otra. El orden de presentación de las tareas fue contrabalanceado.

La administración de las tareas se realizó en un aula tranquila de la misma escuela de los niños y duraba entre 5 y 10 minutos. Una vez sentados, el experimentador le comunicaba al participante que a continuación le explicaría unos cuentos y le haría unas preguntas sobre los personajes. Entonces se administraban las 4 tareas, de juego simbólico o de engaño. Después de explicar cada historia, se hacían 2 preguntas de memoria, para asegurar que el niño la había comprendido. Luego, se hacían 3 preguntas test, donde experimentador focalizaba la atención de los participantes en el Dibujo 2. Las preguntas eran las siguientes:

\section{Pregunta 1. Emoción externa del protagonista:}

Pregunta test 1.1: ¿Qué cara pone Marta, de contenta o triste? Pregunta test 1.2: ¿Por qué pone cara de contenta / triste?

Pregunta 2. Emoción interna del protagonista:

Pregunta test 2.1: ¿Cómo está Marta por dentro, contenta o triste?

Pregunta test 2.2: ¿Por qué está contenta / triste?

Pregunta test 2.3: ¿Está contenta / triste de verdad?

Pregunta test 2.4: ¿Cómo está en realidad?

Pregunta test 2.5: ¿Por qué?

Pregunta 3. Creencias del observador sobre la emoción del protagonista:
Pregunta test 3.1: ¿Juan piensa que Marta está contenta o piensa que está triste?

Pregunta test 3.2: ¿Por qué piensa que está contenta / triste?

Se consideraba que el participante mostraba una comprensión AR emocional si atribuía al protagonista una emoción interna distinta de la emoción externa que éste mostraba en el Dibujo 2. Cabe resaltar que si al preguntar por la emoción interna (pregunta 2.1) el participante no hacía la distinción AR emocional, se le daba otra oportunidad, preguntándole si el protagonista estaba contento o triste de verdad (pregunta 2.3). Si el participante respondía que sí, entonces se pasaba a la siguiente pregunta (pregunta test 3.1), pero si el sujeto respondía que el protagonista no estaba contento de verdad, entonces se le preguntaba cómo estaba el protagonista en realidad y por qué (preguntas 2.4 y 2.5). Si en esta segunda oportunidad el sujeto atribuía una emoción interna distinta a la externa, entonces también se consideraba que había mostrado una comprensión de la distinción AR emocional. Además, se debe resaltar que siempre se daban dos opciones de respuesta para facilitar al máximo la tarea a los niños pequeños: contento o triste.

En relación con la obtención de los datos de las oraciones completivas, se grabaron, transcribieron y analizaron las transcripciones de todas las respuestas de los participantes a las preguntas 1.2, 2.2, 2.5 y 3.2. En total, se analizaron 4044 respuestas.

Se consideraron oraciones completivas aquellas oraciones que seguían la siguiente estructura: verbo de proceso mental, comunicativo o de comportamiento + que + verbo conjugado en indicativo (presente o pasado). A partir de esta definición, los investigadores que aparecen como primer y como segundo autor del artículo clasificaron todas las respuestas de los niños en función de si contenían o no una oración completiva. Del total de 4044 respuestas hubo un $96.19 \%$ de acuerdos (Kappa de Cohen $=0.9038$ ). Además, se estableció una clasificación de las oraciones completivas en función del tipo de verbo en el que estaba incrustado el complemento. Esta clasificación se realizó a partir de la propuesta de clasificación verbal de la base de datos sintácticos del español del proyecto ADESSE (ver, por ejemplo, Albertuz, 2007). De éste modo, se clasificaron las oraciones completivas utilizadas por los niños en las categorías siguientes: cognitivas, comunicativas, perceptivas y de sensación (incluyen las volitivas), en función del verbo en el cual estaban incrustadas (ver apéndice 1).

\section{Puntuación}

Se analizó qué participantes escogieron para el protagonista una emoción interna distinta de la emoción externa en, al menos, una de las cuatro tareas. Por otro lado, se analizó el número de participantes que utilizaron como mínimo una oración completiva en alguna de sus respuestas y los que no. Finalmente, se clasificaron los participantes entre los que uti- 
lizaron alguna oración completiva de cognición, de comunicación, de percepción o de sensación, y los que no.

\section{Resultados}

En este apartado se analizarán, los siguientes aspectos: la comprensión AR emocional, el uso de oraciones completivas, y la relación entre ambos. En estos análisis se han considerado como variables independientes la condición (juego y engaño) y la edad.

\section{Comprensión AR emocional ${ }^{1}$}

En primer lugar se contabilizó el número de participantes que realizaron la distinción AR emocional en, al menos, una tarea (ver Tabla 1). Dado que la variable dependiente (comprensión AR emocional en alguna tarea) era dicotómica, se usó la prueba Chi-cuadrado. Los resultados mostraron un aumento significativo de dicha comprensión en función de la edad, tanto en la condición de juego $\left(\chi^{2}(1, N=169)=\right.$ $81.671, p=.000)$ como en la condición de engaño $\left(\chi^{2}(1, N\right.$ $=168)=101.925, p=.000)$. Al realizar la comparación entre ambas condiciones, se encontraron diferencias significativas a la edad de 6 años $\left(\chi^{2}(1, N=73)=4.112, p=.043\right)$ a favor de la condición de engaño, pero no en el resto de grupos de $\operatorname{edad}(p>$.05).

Tabla 1. Porcentaje de participantes que realizaron la distinción AR emocional en alguna tarea en función de la edad y de la condición.

\begin{tabular}{llllll}
\hline & $\mathbf{4}$ años & $\mathbf{6}$ años & $\mathbf{8}$ años & $\mathbf{1 0}$ años & $\mathbf{1 2}$ años \\
\hline Juego & $22 \%$ & $62 \%$ & $94 \%$ & $100 \%$ & $100 \%$ \\
Engaño & $13 \%$ & $83 \%$ & $94 \%$ & $97 \%$ & $100 \%$ \\
\hline
\end{tabular}

\section{Uso de oraciones completivas}

En este apartado se analizan los datos de los participantes que utilizaron alguna oración completiva en alguna de las tareas, primero a nivel general, y después en función del tipo de oración completiva utilizada.

Para analizar los resultados de los participantes que utilizaron, al menos, una oración completiva en alguna de las tareas (ver Tabla 2) se utilizó la prueba Chi-cuadrado, dado que la variable dependiente (uso o no de completivas) era dicotómica. Los resultados mostraron que no hay diferencias significativas en función de la condición en la cantidad de participantes que han utilizado, al menos, una oración completiva $(\phi>.05)$. Por otra parte, se observó que con la edad aumentaba el número de participantes que utilizaron, al menos, una oración completiva, tanto en la condición de juego $\left(\chi^{2}(4, N=169)=52.301, p=.000\right)$ como en la condición

\footnotetext{
${ }^{1} \mathrm{El}$ análisis de esta comprensión se encuentra analizado en detalle en el artículo de Sidera, Serrat, Rostan, y Sanz-Torrent (2011). Para el presente trabajo solamente se han analizado los participantes que han superado, al menos, una de las cuatro tareas de AR emocional, ya que interesaba saber qué participantes podían comprender la simulación emocional y no en cuantas tareas podían hacerlo.
}

de engaño $\left(\chi^{2}(4, N=168)=49.040, p=.000\right)$. Se observó también que todos los participantes de 10 y 12 años utilizaron alguna oración completiva en la condición de juego, y casi todos en la de engaño.

Tabla 2. Porcentaje de participantes que utilizaron alguna oración completiva en función de la edad y de la condición.

\begin{tabular}{llllll}
\hline & 4 años & $\mathbf{6}$ años & $\mathbf{8}$ años & $\mathbf{1 0}$ años & $\mathbf{1 2}$ años \\
\hline Juego & $44 \%$ & $77 \%$ & $94 \%$ & $100 \%$ & $100 \%$ \\
Engaño & $34 \%$ & $69 \%$ & $88 \%$ & $97 \%$ & $94 \%$ \\
\hline
\end{tabular}

$\mathrm{Al}$ considerar los participantes que utilizaron los distintos tipos de oraciones completivas en, al menos, una ocasión (ver Tabla 3), se observó que, en la condición de juego, existe un aumento significativo en función de la edad de los participantes que utilizaron, al menos, una oración completiva de cognición $\left(\chi^{2}(4, N=169)=52.569, p=.000\right)$, pero no para los otros tipos de oraciones $(p>.05)$.

En cuanto a la condición de engaño, este aumento en función de la edad fue significativo para las oraciones completivas de cognición, $\left(\chi^{2}(4, N=168)=47.437, p=.000\right)$, de comunicación $\left(\chi^{2}(4, N=168)=16.094, p=.003\right)$ y de percepción $\left(\chi^{2}(4, N=168)=12.894, p=.012\right)$, pero no para las de sensación $(\not>.05)$.

Considerando la utilización de algún tipo de oración completiva con verbos comunicativos o cognitivos (ver Tabla 4), se halló un aumento en la utilización de alguno de estos tipos de verbos en función de la edad de los participantes, tanto en la condición de juego $\left(\chi^{2}(4, N=169)=\right.$ 38.922, $p=.000)$ como en la condición de engaño $\left(\chi^{2}(4, N\right.$ $=168)=9.521, p=.049)$. Comparando las dos condiciones, se halló una diferencia significativa en la utilización de estos tipos de verbos a favor de la condición de juego, a los $8,10 \mathrm{y}$ 12 años de edad (8 años: $\chi^{2}(1, N=64)=7.729, p=.005 ; 10$ años: $\chi^{2}(1, N=72)=16.087, p=.000 ; 12$ años: $\chi^{2}(1, N=$ $64)=15.150, p=.000)$ pero no en los otros grupos de edad $(p>.05)$.

\section{Contingencia de utilización de cada tipo de oración completiva y la comprensión AR emocional}

A continuación se analizará la contingencia entre la realización de la distinción AR emocional en alguna tarea y la utilización de alguna oración completiva en función del tipo de verbo (ver Tabla 5). A través de la prueba Chi-cuadrado se observó una relación de contingencia significativa entre la realización correcta de alguna tarea de AR emocional y la utilización de, al menos, una oración completiva de cognición $\left(\chi^{2}(1, N=337)=71.247, p=.000\right)$ o de percepción $\left(\chi^{2}(1, N=337)=5.564, p=.018\right)$, así como una relación cercana a la significación en la utilización de, al menos, una oración completiva de comunicación $\left(\chi^{2}(1, N=337)=\right.$ $3.387, p=.066)$. No hubo ninguna relación significativa con los verbos de sensación $(p>.05)$. 
Tabla 3. Porcentaje de participantes que utilizaron alguna oración completiva en función del tipo de oración, de la edad y de la condición.

\begin{tabular}{ccccccccccc}
\hline & \multicolumn{2}{c}{$\mathbf{4}$ años } & \multicolumn{2}{c}{$\mathbf{6}$ años } & \multicolumn{2}{c}{$\mathbf{8}$ años } & \multicolumn{1}{c}{$\mathbf{1 0}$ años } & \multicolumn{1}{c}{$\mathbf{1 2}$ años } \\
\cline { 2 - 11 } & Juego & Eng & Juego & Eng & Juego & Eng & Juego & Eng & Juego & Eng \\
\hline Sensación & 0 & 12.5 & 0 & 5.6 & 0 & 15.6 & 0 & 33.3 & 0 & 31.3 \\
Percepción & 3.1 & 0 & 2.8 & 5.6 & 6.3 & 15.6 & 5.6 & 13.9 & 6.3 & 15.6 \\
Comunicación & 3.1 & 18.8 & 21.6 & 30.6 & 23.1 & 53.1 & 12.5 & 58.3 & 23.1 & 53.1 \\
Cognición & 40.6 & 9.4 & 70.3 & 58.3 & 93.8 & 71.9 & 97.2 & 75 & 100 & 84.4 \\
\hline
\end{tabular}

Si analizamos los datos en función de la condición, observamos que en la condición de juego, se observó una contingencia significativa entre la distinción AR emocional en alguna tarea y la utilización de alguna oración completiva de cognición $\left(\chi^{2}(1, N=169)=52.424, p=.000\right)$, relación que no fue significativa con los otros tipos de oraciones. En el caso de la condición de engaño, esta relación fue significativa para las oraciones completivas de cognición $\left(\chi^{2}(1, N=\right.$ $168)=52.424, p=.000)$ y de percepción $\left(\chi^{2}(1, N=168)=\right.$ $5.342, p=.021)$, y cercana a la significación para las oraciones completivas de comunicación $\left(\chi^{2}(1, N=168)=3.339\right.$, $p=.068)$.

Tabla 4. Porcentaje de participantes que utilizaron alguna oración completiva con verbos de comunicación o de cognición, en función de la edad y de la condición.

\begin{tabular}{cccccc}
\hline & 4 años & $\mathbf{6}$ años & $\mathbf{8}$ años & 10 años & 12 años \\
\hline Juego & $40.6 \%$ & $67.6 \%$ & $87.5 \%$ & $94.4 \%$ & $93.8 \%$ \\
Engaño & $25 \%$ & $58.3 \%$ & $56.3 \%$ & $52.8 \%$ & $50 \%$ \\
\hline
\end{tabular}

Analizando la contingencia entre la utilización de cada tipo de oraciones completivas en función del grupo de edad se observó que la única relación significativa fue a los 6 años para las oraciones completivas de cognición $\left(\chi^{2}(1, N=73)\right.$ $=4.514, p=.034)$. Así, de los 47 participantes de 6 años que utilizaron alguna oración completiva de cognición, 38 $(80.85 \%)$ realizaron correctamente, al menos, una de las tareas de AR emocional. En cambio, sólo el $57.7 \%$ de los participantes que no utilizaron ninguna oración completiva de cognición fueron capaces de realizar la distinción AR emocional en alguna de las tareas.

Tabla 5. Contingencia entre la comprensión AR emocional y la utilización de alguna oración completiva, según el tipo de verbo, la edad y la condición.

\begin{tabular}{cccc}
\hline & & \multicolumn{2}{c}{$\begin{array}{c}\text { Realiza la distinción AR } \\
\text { emocional en alguna tarea }\end{array}$} \\
\cline { 3 - 4 } $\begin{array}{c}\text { Utiliza alguna } \\
\text { completiva de... }\end{array}$ & & No & Sí \\
\hline \multirow{2}{*}{ Sensación } & No & 74 & 230 \\
& Sí & 4 & 29 \\
\hline \multirow{2}{*}{ Percepción } & No & 77 & 235 \\
& Sí & 1 & 24 \\
\hline \multirow{2}{*}{ Comunicación } & No & 62 & 178 \\
& Sí & 16 & 81 \\
\hline Cognición & No & 53 & 47 \\
& Sí & 25 & 212 \\
\hline
\end{tabular}

$\mathrm{Al}$ analizar la relación entre los participantes que usaron alguna oración completiva con verbos comunicativos o cognitivos (pero no oraciones completivas de otros tipos) con la comprensión de alguna tarea de comprensión AR emocional, se observó una fuerte contingencia entre ambas variables $\left(\chi^{2}(1, N=337)=28.792, p=.000\right)$. Esta contingencia fue significativa tanto en la condición de juego $\left(\chi^{2}(1, \mathrm{~N}=\right.$ $169)=38.334, p=.000)$ como en la de engaño $\left(\chi^{2}(1, N=\right.$ 168) $=5.094, p=.024)$.

A pesar de estas contingencias, se observaron 32 casos en los cuales los participantes, a pesar de no utilizar ninguna oración completiva cognitiva o comunicativa, fueron capaces de realizar la distinción AR emocional en alguna tarea. Además, también hubo 76 casos en los cuales a pesar de utilizar alguna oración completiva cognitiva o comunicativa, los participantes no realizaron la distinción AR emocional en ninguna tarea.

\section{Discusión}

En la primera hipótesis planteada se predecía que algunos participantes serían incapaces de resolver tareas de AR emocional aun siendo capaces de utilizar oraciones completivas con verbos comunicativos o cognitivos. De acuerdo con nuestros resultados, 32 de los participantes fueron incapaces de resolver ninguna tarea de AR emocional aun habiendo utilizado oraciones completivas con verbos cognitivos o comunicativos. Por tanto, se puede afirmar que esta primera hipótesis se ha cumplido. Estos datos van en la misma dirección que los aportados por los estudios que sugieren que la sintaxis de la complementación no es una condición suficiente para la resolución de tareas de falsa creencia (Remmels y Peters, 2009). Sin embargo, se debe resaltar un aspecto importante, y es que las tareas de AR emocional se comprenden más tarde que las tareas de falsa creencia (Wellman y Liu, 2004). Por tanto, solo se puede afirmar que la sintaxis de la complementación no es una condición suficiente para resolver tareas de apariencia realidad. A continuación, se discutirá si es una condición necesaria.

Los resultados obtenidos en el presente estudio muestran que con la edad los niños utilizan más oraciones completivas de comunicación, cognición y percepción. Sin embargo, ¿qué relación existe entre la mejora en esta capacidad y el desarrollo de la capacidad para comprender que las emociones aparentes pueden ser distintas de las emociones reales? En otras palabras, ¿el hecho de que un niño sea capaz 
de dominar la sintaxis de la complementación, garantiza que realice correctamente la distinción AR emocional? En la segunda hipótesis del presente estudio se preveía que los niños que realizaran la comprensión $\mathrm{AR}$ emocional serían capaces de utilizar oraciones completivas de comunicación o de cognición en sus respuestas. Esta hipótesis no se ha cumplido, o solo parcialmente, ya que muchos de los participantes han resuelto las tareas de AR emocional sin utilizar oraciones completivas de ningún tipo. Por ejemplo, en la tarea de engaño que se muestra en el Apéndice 2, donde María pone cara de triste para que su hermana le de un abrazo, al preguntar a los participantes por qué María pone cara triste, algunos niños dieron las siguientes respuestas: "Porque le habia dicho una mentira y no lo sabe su hermana", "Para que su bermana se lo crea", "Para disimular", "Porque le ha dicho una mentira y se la ha creído". De este modo, se puede explicar perfectamente una simulación emocional y la consecuente creencia falsa del observador sin la necesidad de utilizar oraciones completivas. En la misma línea, Cheung et al. (2004) resaltan que en cantonés, la flexibilidad del orden de las frases y las partículas de final de frase proveen a la lengua de formas alternativas para expresar mentiras y errores, y que por tanto, no necesariamente se deben utilizar oraciones completivas. En el caso concreto de las lenguas románicas, cabe la posibilidad de utilizar pronombres con el mismo sentido que las oraciones completivas. Por tanto, existen formas alternativas de expresar la comprensión AR emocional. Estas consideraciones, junto con los datos del presente estudio, sugieren que la habilidad de producir oraciones completivas no es necesaria para la comprensión de la distinción entre las emociones externas y las emociones internas. Por otra parte, no se puede descartar la posibilidad de que la comprensión de oraciones completivas tenga un papel en el desarrollo la comprensión AR emocional, porque en el presente estudio no se ha investigado si los niños comprendían o no las oraciones completivas, sino sólo si las utilizaban o no en sus justificaciones. En este sentido, el tipo de situación que se proponga a los niños para que la expliquen afectará al tipo de respuesta que den, y por tanto, no necesariamente expresarán todo lo que saben. Este aspecto queda reflejado, por ejemplo, a la edad de 4 años, donde la condición de engaño elicitó principalmente oraciones completivas con verbos comunicativos (que parecían intentar explicar la diferencia entre lo que se ha dicho y lo que ha ocurrido realmente), mientras que la condición de juego elicitó mayormente verbos cognitivos (que intentaban explicar lo que el personaje está simulando).

Los resultados de este estudio muestran que, aunque la utilización de oraciones completivas no es suficiente para ayudar a los niños a comprender la distinción AR emocional, sí que existe una relación importante entre ambas. En cuanto al papel de la condición experimental en esta relación, los análisis han mostrado que existe una contingencia entre el uso de las oraciones completivas y la comprensión AR emocional en ambas condiciones. Estos resultados sugieren que el desarrollo de la sintaxis de la complementación podría jugar un papel importante en el desarrollo de la comprensión
AR emocional tanto en situaciones donde el objetivo es engañar a alguien a través de la simulación, como en situaciones en las que el objetivo es jugar a simular emociones. En este aspecto, es importante resaltar que no solo se encontraron diferencias en la utilización de oraciones completivas con verbos cognitivos o comunicativos en función de la condición, sino que además, los tipos de verbos incrustados en las oraciones completivas que se mostraron relacionados con la comprensión AR emocional fueron distintos para ambas condiciones. Estos resultados indican que los distintos tipos de verbos incrustados en las oraciones completivas podrían tener papeles distintos en el desarrollo de la comprensión de que las emociones se pueden simular en función del contexto o de las intenciones al simular emociones. Por otro lado, cabe resaltar que sólo a la edad de 6 años ha existido contingencia entre el uso de, al menos, una oración completiva y la comprensión AR emocional. La ausencia de esta contingencia en los demás grupos de edad no es sorprendente, puesto que hay que tener en cuenta que muy pocos niños de 4 años fueron capaces de realizar la distinción AR emocional y que, al contrario, la mayoría de los niños de 8 años o mayores lo consiguieron. En definitiva, los resultados de este estudio apoyan la propuesta de de Villiers (2007) de que el dominio de la sintaxis de la complementación es una herramienta útil para el razonamiento sobre las falsas creencias, aunque es posible que no sea una herramienta suficiente ni imprescindible.

En relación con el tipo de oraciones completivas utilizadas, se observó una contingencia entre los niños que realizaron correctamente como mínimo una tarea de comprensión AR emocional y los que utilizaron alguna oración completiva de comunicación o cognición y de percepción. Esto no fue así en los verbos de sensación, que incluían básicamente construcciones con verbos volitivos. Por tanto, aunque en la lengua catalana los niños comiencen a realizar construcciones del tipo "voler que" (querer que) alrededor de los 2 años y medio (Aparici et al., 2001), este tipo de construcciones, como mínimo en el presente trabajo, no están vinculadas a la comprensión de la distinción AR emocional. También es destacable la vinculación observada entre las oraciones completivas de tipo perceptivo y la comprensión AR emocional, ya que la literatura previa sólo había destacado la importancia de las completivas de tipo comunicativo y cognitivo en el desarrollo de la TM (por ejemplo, de Villiers, 2005). A partir de los resultados obtenidos en este estudio, sería interesante que la investigación futura indagara en el papel de las oraciones completivas con verbos de tipo perceptivo en la comprensión AR emocional.

\section{Conclusiones}

En resumen, el presente trabajo sugiere que la utilización de oraciones completivas con verbos comunicativos o cognitivos no es una condición ni necesaria ni suficiente para el desarrollo de la comprensión de la distinción entre la apariencia y la realidad emocional. Sin embargo, se ha constatado 
una vinculación importante entre ambas variables, no solamente con oraciones completivas con verbos cognitivos o comunicativos, vinculación ya destacada en la literatura previa (de Villiers, 2005), sino también con verbos perceptivos. Finalmente, se sugiere la necesidad de estudiar la relación entre el desarrollo de la comprensión (y no solo de la utilización) de distintos tipos de oraciones completivas y la comprensión de que las emociones externas pueden diferir de las internas.

\section{Referencias}

Albertuz, F. (2007). Sintaxis, semántica y clases de verbos: Clasificación verbal en el proyecto ADESSE", Cano López, Pablo (Coord): Actas del VI Congreso de Lingüistica General, Santiago de Compostela, 3-7 de mavo de 2004, Vol. 2, Tomo 2, Las lenguas y su estructura (IIb), pp. 2015-2030.

Aparici, M., Serrat, E., Capdevila, M., y Serra, M. (2001). Acquisition of Complex Sentences in Spanish and Catalan Speaking Children. En K. Nelson, A. Aksu-Koç, C. Johnson (eds.), Children's Language, 11 (pp.125). Lawrence Erlbaum: Mahwah (NJ).

Astington, J. W., y Jenkins, J. M. (1999). A longitudinal study of the relation between language and theory of mind development. Developmental Psychology, 35, 1311-1320.

Astington, J. W., y Baird, J. A. (2005). Why language matters for the theory of mind. New York: Oxford University Press.

Bloom, L., Rispoli, M., Gartner, B., y Hafitz, J. (1989). Acquisition of complementation. Journal of Child Language, 16, 101-120.

Bretherton, I., Fritz, J., Zahn-Waxler, C., y Ridgeway, D. (1986). Learning to talk about emotions: A functionalist perspective. Child Development, 57 , 529-548.

Cheung, H., Chen, H.-C., Creed, N., Ng, L., Wang, S. P., y Mo, L. (2004). Relative roles of general and complementation language in theory-ofmind development: Evidence from Cantonese and English. Child Development, 75(4), 1155-1170.

de Villiers, J. G., y Pyers, J. E. (2002). Complements to cognition: a longitudinal study of the relationship between complex syntax and false-beliefunderstanding. Cognitive Development, 17, 1037-1060.

de Villiers, P. A., Burns, F., y Pearson, B. (2003). The role of language in the Theory of Mind development of language-impaired children: complementing theories. In Beachley, B., Brown, A., Conlin, F. (Eds.), Proceedings of the $27^{\text {th }}$ Annual Boston University Conference on Language Development. Cascadilla Press, Somerville, MA, pp. 232-242.

de Villiers, J. G. (2005). Can language acquisition give children a point of view? In: Astington, J., Baird, J. (Eds.), Why Language Matters for Theory of Mind (pp. 186-219). New York: Oxford University Press.

de Villiers, J. G. (2007). The interface of language and Theory of Mind. Lingua, 117, 1858-1878.

de Villiers, P. A., y de Villiers, J. G. (en prensa). Deception dissociates from false belief reasoning in deaf children: Implications for the implicit versus explicit theory of mind distinction. British Journal of Developmental Psychology.

Fisher, N., Happé, F., y Dunn, J. (2005). The relationship between vocabulary, grammar, and false belief task performance in children with autistic spectrum disorders and children with moderate learning difficulties. Journal of child Psychology and Psychiatry, 46, 401-419.

Gross, D., y Harris, P. L. (1988). False belief about emotion: Children's understanding of misleading emotional displays. International Journal of Behavioral Development, 11 (4), 475-488.

Hale, C. M., y Tager-Flusberg, H. (2003). The influence of language on theory of mind: A training study. Developmental Science, 6(3), 346-359.

Hansen, M. B. (2010). If you know something, say something: young children's problem with false beliefs. Frontiers in Developmental Psychology, 1(23), 1-7.

Harris, P. L., Donnelly, K., Guz, G. R., y Pitt-Watson, R. (1986). Children's understanding of the distinction between real and apparent emotion. Child Development, 57, 895-909.
Agradecimientos.- Damos las gracias a todos los niños y niñas entrevistados por su inestimable colaboración en el presente trabajo. También queremos expresar nuestra gratitud a las escuelas y al instituto de la ciudad de Girona que han participado en el estudio: Annexa-Joan Puigbert, Montfalgars, Joan Bruguera, Montjuïch, Dalmau Carles, Verd y IES Santiago Sobrequés. Este trabajo ha sido posible gracias a una ayuda (SING2010B/9) de la Universitat de Girona y también gracias a una beca predoctoral (BRUDG20092013) concedida por la misma universidad.

Harris, P. L. (2005). Conversation, pretense, and theory of mind. En J. W. Astington y J. A. Baird (Eds.), Why language matters for theory of mind (pp. 70-83). Oxford, UK: Oxford University Press.

Jackendoff, R. (1996). How language helps us think. Pragmatics and Cognition, 4, 1-31.

Katis, D., y Stampouliadou, C. (2009). The development of sentential complement constructions in Greek: evidence from a case study. Constructions and Frames, 1 (2), 222-261.

Kidd, E., Lieven, E., y Tomasello, M. (2005). The acquisition of complement clause constructions: A sentence repetition study. Proceedings of the 32nd Stanford Child Language Forum, 50-59.

Kidd, E., Lieven, E., y Tomasello, M. (2006). Examining the role of lexical frequency in the acquisition and processing of sentential complements. Cognitive Development 21, 93-107.

Limber, J. (1973). The genesis of complex sentences. In T. E. Moore (Ed.), Cognitive development and the acquisition of language (pp. 169-185). New York, NY: Academic Press.

Lind, S. E. y Bowler, D. M. (2009). Language and theory of mind in autism spectrum disorder: The relationship between complement syntax and false belief task performance. Journal of Autism and Developmental Disorders, 39 (6), 929-937.

Lohmann, H., y Tomasello, M. (2003). The role of language in the development of false belief understanding: A training study. Child Development, 74, 1130-1144.

Low, J. (2010). Preschoolers' implicit and explicit false-belief understanding: relations with complex syntactical mastery. Child Development, 81 (2), 597-615

Miller, C. A. (2004). False belief and sentence complement performance in children with specific language impairment. International Journal of Language \& Communication Disorders, 39 (2), 191-213.

Miller, C. A. (2006). Developmental relationships between language and theory of mind. American Journal of Speech-Language Pathology, 15, 142-154.

Miller, C. A., y Verrilli, S. (2005). Case studies of sentence complement training and false belief performance. Contemporary Issues in Communication Sciences and Disorders, 32, 43-52.

Milligan, K., Astington, J. W., y Dack, L. A. (2007). Language and theory of mind: Meta-analysis of the relation between language ability and falsebelief understanding. Child Development, 77, 622-646.

Papafragou, A., Cassidy, K., y Gleitman, L. (2007). When we think about thinking: The acquisition of belief verbs. Cognition 105, 125-165.

Paynter, J., y Peterson, C. (2010). Language and ToM development in autism versus Asperger syndrome: Contrasting influences of syntactic versus/semantic maturity. Research in Autism Spectrum Disorders, 4 (3), 377 385.

Perner, J., Sprung, M., Zauner, P., y Haider, H. (2003). Want-that is understood well before think-that, say-that, and false belief: A test of de Villiers' linguistic determinism on German speaking children. Child Development, 74, 179-188.

Pons, F., Harris, P. L., y de Rosnay, M. (2004). Emotion comprehension between 3 and 11 years: Developmental periods and hierarchical organizations. European Journal of Developmental Psychology, 1, 127-152.

Resches, M., Serrat, E., Rostan, C. y Esteban, M. (2010). Lenguaje y teoría de la mente: una aproximación multidimensional. Infancia y Aprendizaje, 33 (3), 315-333. 
Segal, G. (1998). Representing representations. En P. Carruthers y J. Boucher (Eds.), Language and thought: Interdisciplinary themes (pp. 146-161). New York: Cambridge University Press.

Serrat, E., Rostan, C., Vallès-Majoral, E., Esteban, M., Sidera, F., y Serrano, J. (en prensa) Labelling improves false belief understanding. A training study. Spanish Journal of Psychology.

Sidera, F., Serrat, E., Rostan, C., y Sanz-Torrent, M. (2011). Do Children Realize That Pretend Emotions Might Be Unreal? The Journal of Genetic Psychology: Research and Theory on Human Development, 172, 40-55.

Schick, B., de Villiers, P., de Villiers, J., y Hoffmeister, R. (2007). Language and theory of mind: A study of deal children. Child Development, 78, 376396.

Slade, L. y Ruffman, T. (2005). How language does (and does not) relate to theory of mind: A longitudinal study of syntax, semantics, working memory and false belief. British Journal of Developmental Psychology, 23, 126.

Stephens, J. (2006). Longer-term aspects of the language development of children with cochlear implants. Audiological Medecine, 4, 151-163.

Tager-Flusberg, H. y Joseph, R. (2005). How language facilitates the acquisition of false belief in children with autism. En J. Astington y J. Baird (Eds.), Why Language Matters for Theory of Mind (298-318). New York: Oxford University Press.

Wellman, H. M., Cross, D., y Watson, J. (2001). Meta-analysis of theory of mind development: The truth about false belief. Child Development, 72, 655-684.

Wellman, H. M. y Liu, D. (2004). Scaling of theory-of-mind tasks. Child Development, $75,523-541$.

(Articulo recibido: 24-3-2011, revisado: 16-3-2012, aceptado: 25-3-2012)

Apéndice 1. Clasificación de las oraciones completivas según el tipo de verbo en el cual estaban incrustadas (verbos en catalán).

\begin{tabular}{|c|c|c|c|c|}
\hline \multicolumn{2}{|c|}{ Verbos de cognición } & Verbos de sensación & Verbos de percepción & Verbos de comunicación \\
\hline Adonar-se que & Fer veure que & Estar content que & Descobrir que & "Contar" que \\
\hline Confiar en que & Imaginar-se que & Voler que & Enxampar que & Dir que \\
\hline Creure que & Inventar-se que & & Mirar que & Explicar que \\
\hline Creure's que & Jugar a que & & Notar-se que & Fer una mentida que \\
\hline Donar-se compte & Pensar que & & Veure que & Mentir que \\
\hline Enganyar que & Pensar-se que & & Veure's que & \\
\hline Esperar que & Posar-se en el paper de que & & Voler semblar que & \\
\hline Fer broma que & Saber que & & Voler veure que & \\
\hline Fer com que & Semblar que & & & \\
\hline Fer compte que & Sospitar que & & & \\
\hline Fer creure que & Voler enganyar que & & & \\
\hline Fer mentida que & Voler fer creure & & & \\
\hline Fer que & Voler fer que & & & \\
\hline Fer un joc que & Voler fer veure & & & \\
\hline
\end{tabular}

\section{Apéndice 2. Ejemplo de una tarea de juego simbólico}

Dibujo 1: Marta y Juan están jugando a médicos. Están contentos porque se lo pasan bien.

Dibujo 2: Para jugar, Marta dice: “Venga Juan, vamos a jugar a que me he hecho daño en la pierna. ¡Uy que daño!”, y Juan dice: "Vale, pues yo te curaré. ;Tranquila bonita!"

\section{Ejemplo de una tarea de engaño}

Dibujo 1: Imma cae al suelo. Está contenta porqué no se ha hecho daño.

Dibujo 2: Después llega su hermana. Imma quiere que su hermana piense que se ha hecho daño, porque así le dará un abrazo, y le dice una mentira. Le dice: "Hermanita, me he hecho mucho daño en la pierna". 